\title{
Support of Nuclear Engineering Education and Research at the University of Michigan
}

\author{
Progress Report for the Period \\ May 15, 1992 - May 14, 1993
}

\author{
Project Director \\ William R. Martin \\ Department of Nuclear Engineering \\ The University of Michigan \\ Ann Arbor, Michigan 48109-2104

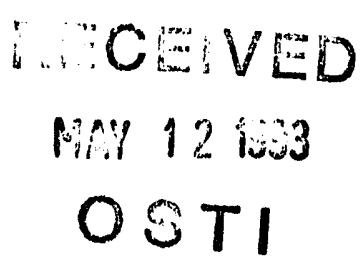

March, 1993

This report was prepared as an account of work sponsored by the United States Government. Neither the United States nor the Department of Energy, nor any of their employees, nor any of their contractors, subcontractors, or their employees, makes any warranty, express or implied, or assumes any legal liability or responsibility for the accuracy, coinpleteness, or usefulness of any information, apparatus, product or process disclosed or represents that its use would not infringe privately-owned rights.

Prepared for

THE U. S. DEPARTMENT OF ENERGY

GRANT NO. DE-FG02-92ER-75728 


\title{
Support of Nuclear Engineering Education and Research at the University of Michigan
}

\begin{abstract}
This report describes progress on four different projects in the fission reactor area that have been supported by the grant during the past year. These projects are:

- Accelerator transmutation of nuclear waste (Steve Pearson)

- Neutronic analysis of the Ford Nuclear Reactor (Brent Renkema)

- Developing Monte Carlo benchmarks for commercial LWR configurations (Jie Du)

- Monte Carlo depletion capability for massively parallel processors (Amit Majumdar)
\end{abstract}

These tasks are briefly described and progress to date is presented.

\section{Background}

Commonwealth Edison Company (CECo) took the initiative in 1991 to establish a program to support nuclear engineering education at a selected number of universities, including the University of Michigan (UM), and the Department of Energy (DOE) has agreed to match these funds. The UM Department of Nuclear Engineering (UMNE) proposed a graduate student research program in conventional fission reactor engineering. The research program was designed to complement existing research programs and leverage existing funds already committed to research in conventional fission reactor engineering. A separate effort supported directly by CECo inclured funding for undergraduate scholarships, graduate traineeships, equipment, and other initiatives, focusing more on improving the instructional environment within the department as well as creating a number of scholarships to encourage increased enrollment. 


\section{Description of Progress}

The current project has supported graduate students (given in parentheses) working in the following areas related tc fission reactor engineering:

- Accelerator transmutation of nuclear waste (Steve Pearson)

- Neutronic analysis of the Ford Nuclear Reactor (Brent Renkema)

- Developing Monte Carlo benchmarks for commercial LWR configurations (Jie Du)

- Monte Carlo depletion capability for massively parallel processors (Amit Majumdar)

Progress on each of these tasks will be described in the following paragraphs.

\section{Assessment of the accelerator transmutation of nuclear waste concept}

As part of the study on handling high-level radioactive wastes, there has been renewed interest in separation and transmutation of long-lived actinides. Although it has been generally assumed that transuranic actinides could be efficiently used primarily in fast-spectrum nuclear reactors, e.g., liquid-metal cooled reactors (LMRs), the possibility of transmuting transuranics in accelerator-driven thermal-spectrum facilities is being studied by Los Alamos National Laboratory. The effectiveness of recycling transuranics, including plutonium isotopes, in light water reactors (LWRs) is also under investigation.

Stephen Pearson has begun to evaluate the feasibility and merits of the Accelerator Transmutation of Nuclear Waste (ATW) concept proposed by Los Alamos. He uses the ORIGEN isotopics depletion code to evaluate the impact of different neutron flux spectra on the equilibrium transuranics composition and depletion rates of long-lived actinides. Although the ORIGEN code is limited to point depletion calculations, Stephen will use a number of cross section libraries representing different flux spectra and perform parametric studies to compare the high-intensity thermai spectrum of the ATW concept with typical LMR and LWR spectra. As time allows, he also would like to study the optimal processing frequency of the actinide blankets in the ATW concept. 


\section{Neutronic analysis of the Ford Nuclear Reactor}

Effort has been underway to improve and enhance the neutronic analysis methodology for characterization of the Ford Nuclear Reactor (FNR) core in support of the irradiation of pressure vessel samples. The project began in 1991 with the incore irradiation of Yankee Rowe pressure vessel samples and supporting neutronic analysis for the determination of fission source distributions throughout the core. Brent Renkema began to participate in this project during the summer of 1992, shortly after the steel samples were loaded in the water reflector under the Heavy-Section Steel Irradiation (HSSI) Program. The HSSI Program is a long-term materials irradiation program administered by Oak Ridge National Laboratory and the excore irradiation of steel capsules in the FNR core is still continuing.

Brent performed two- and three-dimensional diffusion theory calculations to obtain power distributions for FNR cycles representative of the irradiation period for the HSSI Program. $\mathrm{He}$ also assisted in the generation of effective two-group constants for the heavy water tank so that the fast neutron albedo, at the core-heavy water interface, determined from transport theory calculations is preserved in diffusion theory calculations. With this improved representation of the heavy water reflector, and other modifications and enhancements in the lattice physics and global diffusion theory methodology, calculated thermal flux distributions agree with selfpowered neutron detector measurements generally within $\pm 10 \%$. Brent also made significant contributions in performing a number of parametric studies, which were valuable in assessing the accuracy of our power distribution calculations. Brent is currently performing a sequential simulation of a number of FNR cycles, with a fine-mesh two-dimensional representation of the whole core, in an effort to assess the impact of inaccuracies in assembly burnup distributions estimated by the FNR staff. The study will cover approximately 40 cycles, with actual fuel loading patterns and operating history explicitly represented. Brent will also participate in calculating effective sub-cadmium cross sections for wires used for flux mapping in the FNR core. 


\section{Developing Monte Carlo benchmarks for commercial LWR configurations}

An agreement has recently been negotiated with Studsvik of America to allow the UM to have their commercial reactor analysis codes SIMULATE-3 and CASMO-3 for research and instructional purposes. However, there a number of reactor configurations for which adequate benchmarks are not available to verify the predictions of these codes, primarily lattices with high plutonium loadings which are particularly difficult to handle. These regions can occur in BWRs which are operated to maximize the conversion of fertile uranium to plutonium, and represent an area of uncertainty in reactor analysis. The goal is to develop several benchmark configurations which will be analyzed by the MCNP Monte Carlo code from Los Alamos, which is also resident on the UM workstations. At this point, we have MCNP running on our system and Jie Du is familiarizing himself with the code and with using CASMO-3 and SIMULATE-3. Benchmark configurations are being selected with the concurrence of Studsvik of America, and we expect comparisons to be made this summer on a few configurations.

\section{Monte Carlo depletion capability for massively parallel processors}

The accuracy and robust nature of Monte Carlo codes for reactor applications is well-known by reactor analysts. The most complicated geometry, as long as it can be represented by mathematical surfaces, and the most complicai id material composition, as long as cross sections are known or can be modeled, can be modeled with Monte Carlo codes such as MCNP. The historical impediment to the widespread use of Monte Carlo has been the exorbitant demands on computer time and memory to analyze realistic configurations, such as full-core reactor models, or even assembly models for that matter. This is especially true for depletion analyses, which are traditionally done with simple depletion models which assume either a fixed power or fixed scalar flux during a time step. Even if Monte Carlo is used for the within-time step problem, a simple timestepping is always done to deplete the configuration. With the advent of massively parallel processors (MPPs) in recent years, it is reasonable to ask if Monte Carlo depletion is 
possible, using Monte Carlo to simulate the time-dependent behavior as well as the usual spacedependent behavior. Amit Majumdar is examining this topic and has been using the 32 processor Kendall Square Research KSR-1 computer that the UM recently acquired. He has adapted a prototype time-dependent Monte Carlo code to the KSR-1 and has run it successfully on from one to 28 processors, with the 28 processor case yielding a speedup of 24 . These results are being finalized and will be written up in the next progress report. The next step is to look at the time algorithm for the Monte Carlo simulation and examine different approaches for doing the isotopic depletion. 
